\title{
Proof of Conceptual Study on Alternate Binders
}

\author{
Prathyusha Yadali ${ }^{1}, \mathrm{R}$ Selvaraj ${ }^{2}$, Neeraja $\mathrm{D}^{3}$ \\ ${ }^{1}$ Post Graduate Student, VIT University, Vellore, Tamil Nadu, India \\ ${ }^{2}$ Principal Scientist, CSIR-Central Electro-Chemical Research Institute, Karaikudi, Tamil Nadu, India \\ ${ }^{3}$ Associate Professor, VIT University, Vellore, Tamil Nadu, India
}

\begin{abstract}
Concrete is most widely used construction material in the world next to water. It is estimated that every day around $3 \mathrm{~km}^{3}$ of concrete is cast globally. Besides, manufacture of cement is highly energy intensive, consumes voluminous natural resources and causes environmental health hazards. In addition, it produces considerable amount of green-house gases like $\mathrm{CO}_{2}$. Apparently, this is the cause of concern among the researchers global-wide regarding cement, which is impelling them to develop alternate materials that could replace cement completely. Keeping this essential need in mind, an attempt is made in this paper to formulate and study the proof of concept of the binder properties by considering 6 different groups of binders, namely, phosphates, carbonates, Magnesium Oxide, Di-Ammonium Phosphate, Poly Vinyl Alcohol and Geo-Polymer. Conclusions are drawn on their suitability as promising alternate binders for future concrete.
\end{abstract}

Keywords: Alternate binder, phosphates, Carbonates, Magnesium Oxide, Di-Ammonium Phosphate, Poly Vinyl Alcohol and Geo-Polymer, Fly Ash, Clay, Borax.

\section{Introduction}

Cement is an important constituent material in concrete which is used in all types of civil engineering works. India is the second largest cement manufacturing nation next to China. Cement Concrete is most widely used,sinceit can be cast to any shape and size with and without steel reinforcements. Cementitious materials have played a major role in structural construction since ancient times, in all the civilizations [1-2]. Egyptians used calcined gypsum as cement, Romans and Greeks used lime to make mortar. Romans found that cement could be made that can set under water and applied this in construction of harbors. And, cement was made by adding crushed volcanic ash to lime and was later called pozzolanic cement, named after the village of Pozzuoli near Vesuvius. Britain used crushed brick and tile, where volcanic ash was scarce [3]. During Industrialization phase of history, there was a huge demand of newer materials to suit various industrial requirements. Joseph Aspdin acquired patent in 1824 for Portland cement, named after Portland stone, a widely used construction stone in England at that time. But his cement was not that strong as it was not heated at optimum temperatures. In 1845, Isaac Johnson made the first modern Portland cement with the existing materials after calcination at higher temperature ranges from $1400^{\circ} \mathrm{C}-1500^{\circ} \mathrm{C}[4-7]$.

Cement has definitely revolutionized the construction industry but it has its fair share in environmental degradation and global greenhouse effect. Cement industry primarily produces $\mathrm{CO}_{2}$, a major green-house gas. It contributes up to $5 \%$ of worldwide man-made emissions; in which $50 \%$ attributes to chemical processes and the rest accounts for burning of fuel.

There has been a growing interest since past century in reduction of $\mathrm{CO}_{2}$ emissions. With respect to this, there have been a lot of debates of late on global scale and also special emphasize was made to develop alternate binders that could replace OPC completely. And thus, this subject of research in alternate binders is finding the need of the ever. In addition, utilization of industrial by-products like Fly Ash, sand, micro-silica, lime powder, silica powder, Quartz powder(QP), natural pozzolana like clay are to be utilized in manufacture of Alternate binders[8-10].This is essentially required because manufacture of OPC requires high quantum of natural resources, it is high energy intensive and causes land and air pollution which leads to health hazards like lung function impairment, chronic lung diseases, carcinoma of lungs, stomach and colon, Silicosis.

Considering all the above mentioned facts, it is inevitable to develop alternate binders with low energy intensive, eco-friendly nature, economical with good affordability without compromising strength.

Alternate Binders are the materials other than cement which react with Fine Aggregate, Coarse Aggregate, Pozzolanic materials, and other additives to form a hard mass.

Binders can be classified into 2 categories: Hydraulic Binders and Non-hydraulic binders. In case of Hydraulic binders, water is used for mixing of constituents of concrete, it sets and hardens with hydration process. In case of Non-hydraulic binders, the constituent materials are mixed together to form a solid mass by chemical reaction i.e.; chemical cross-linking [11, 12]. In general, Non- hydraulic binders produce high strength materials without water curing. As this setting takes hardly a day, these alternate non-hydraulic binders are highly useful for manufacture of pre-cast concrete elements, repair works and mass concreting with enhanced durability and mechanical strength. 
A very little amount of published research work is available on Alternate Binders.

The present work comprises of formulating and evaluating 6 different kinds of mortars with different binders which will be discussed later.

\section{Experimental Study:}

6 different sets of families of binders namely:

1. Phosphate based

2. Carbonate based

3. Magnesium Oxide $(\mathrm{MgO})$ based

4. Di-Ammonium Phosphate based

5. Geo Polymer based

6. Poly Vinyl Alcohol based are developed with different filler materials such as Fly Ash, clay, sand, brick powder and Alumina clay.

Phosphate and Di-Ammonium Phosphates are purchased from local market. Sodium silicate was purchased from local chemical suppliers. Magnesium oxide, Sodium Carbonate, Calcium Carbonate, Poly Vinyl Alcohol, Magnesium Sulphate, Magnesium chloride were purchased from HiMedia Laboratories Pvt. Ltd; Mumbai. Clay, Sand and brick powder were obtained locally. Fly Ash was collected from Tuticorin Thermal Energy Power plant. Fineness modulus of sand used was 1.93. Potable tap water was used for mixing of materials.

PVC cylindrical moulds were prepared using PVC pipe of $83 \mathrm{~mm}$ internal diameter and $25 \mathrm{~mm}$ thickness with single vertical split for de-moulding purpose. 33 combinations were prepared as indicated in Table 1 using various binders mentioned earlier. The mixes prepared are cast into the PVC cylinder moulds and finished after compaction. After $24 \mathrm{hrs}$, cast specimens are de-moulded and are found to be suitable for making concrete .The observations are tabulated in Table1.

Fig 1 to Fig 7 shows various categories of different families of binders used.

Fig 1
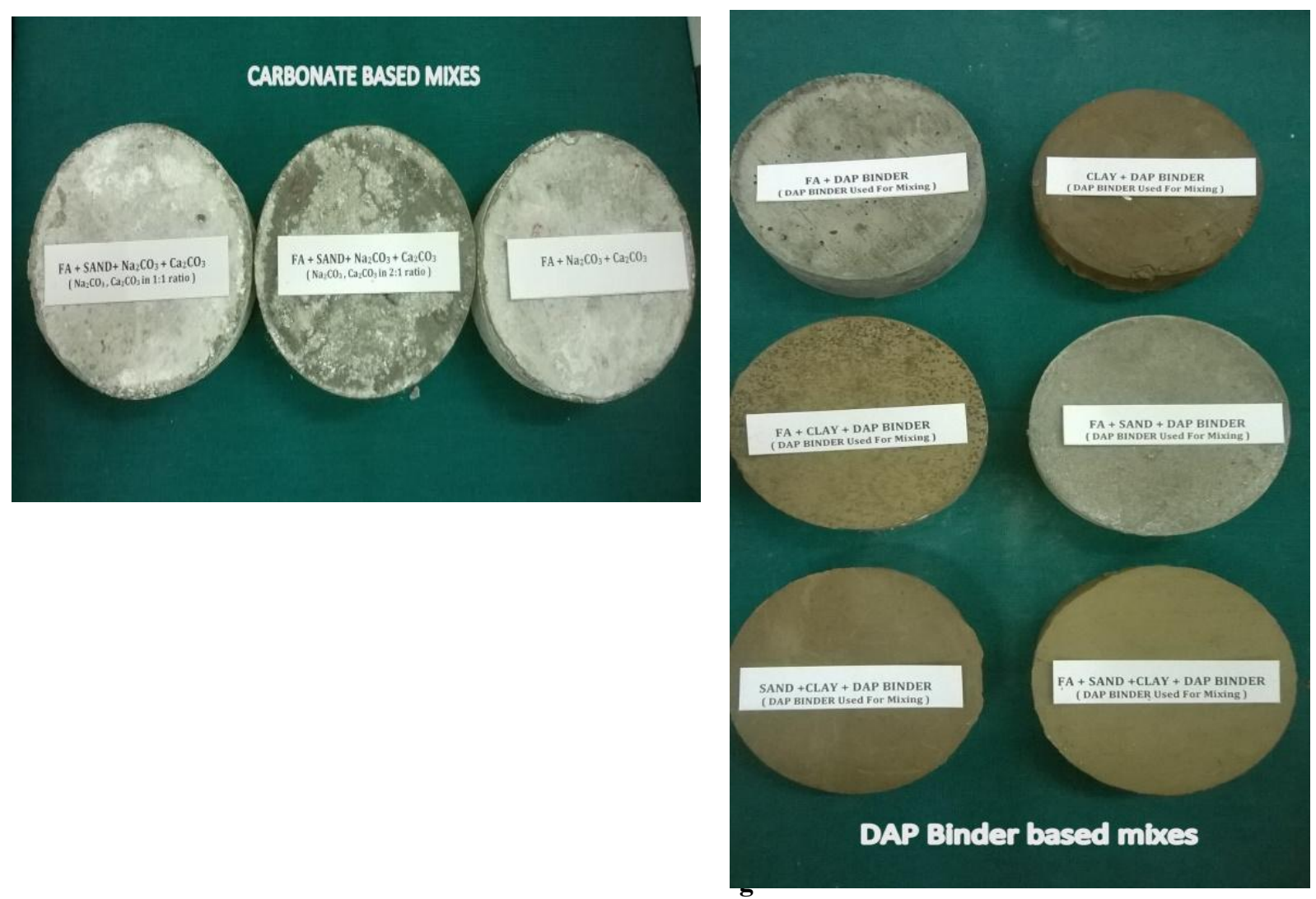

2 


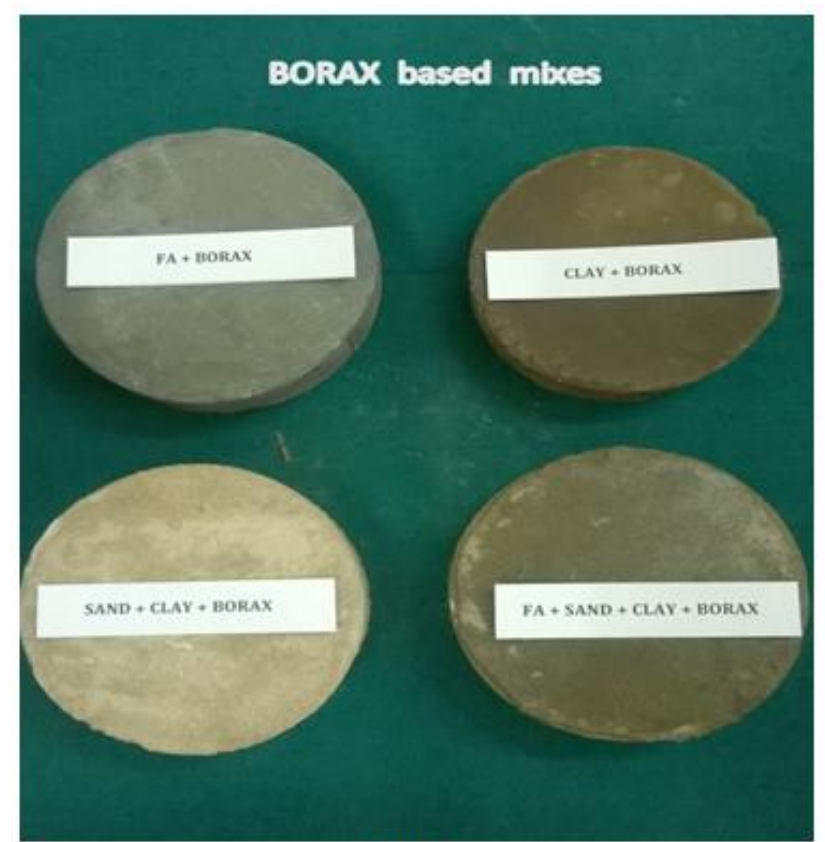

Fig 3

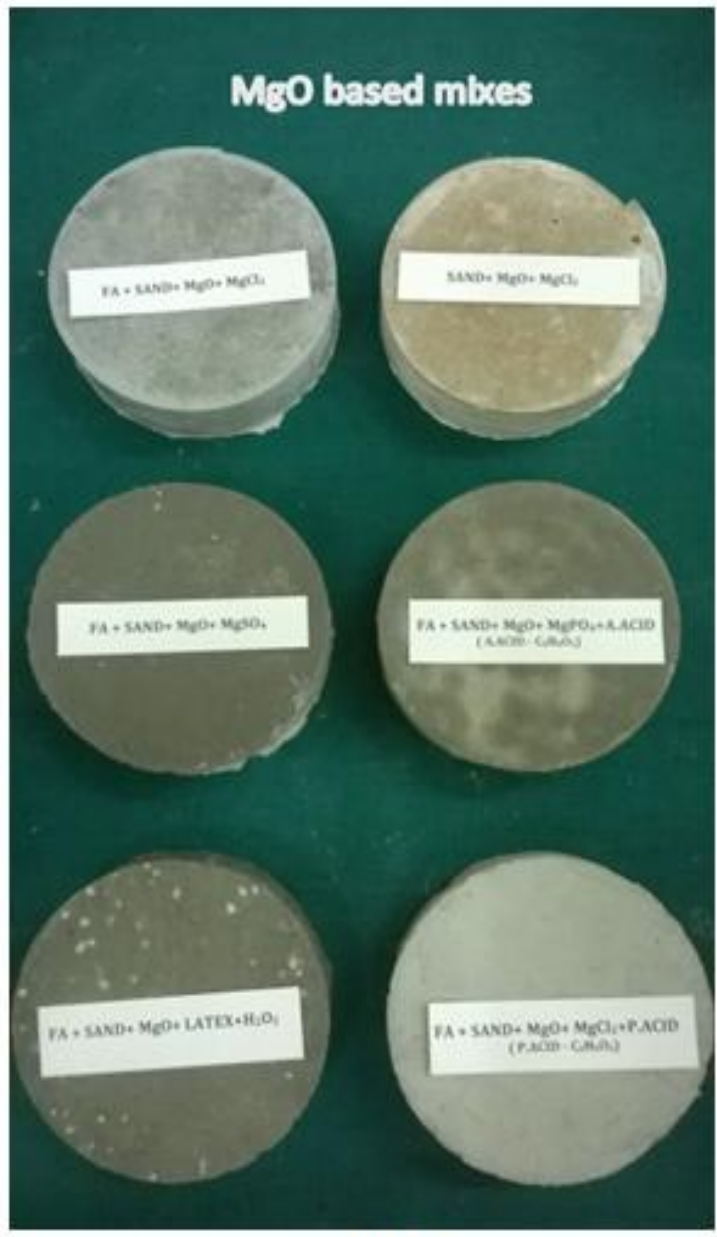

Fig 5

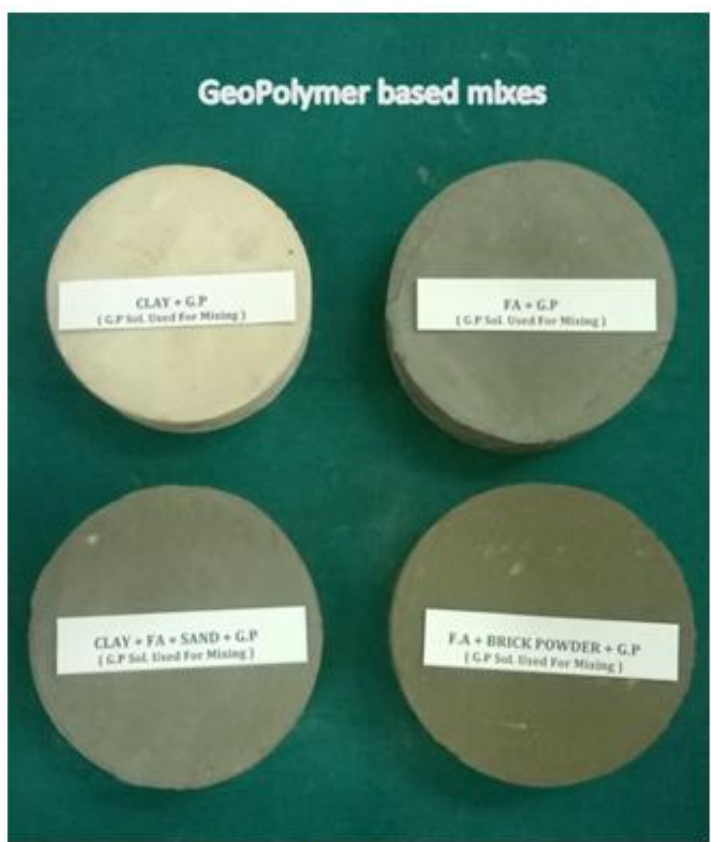

Fig 4

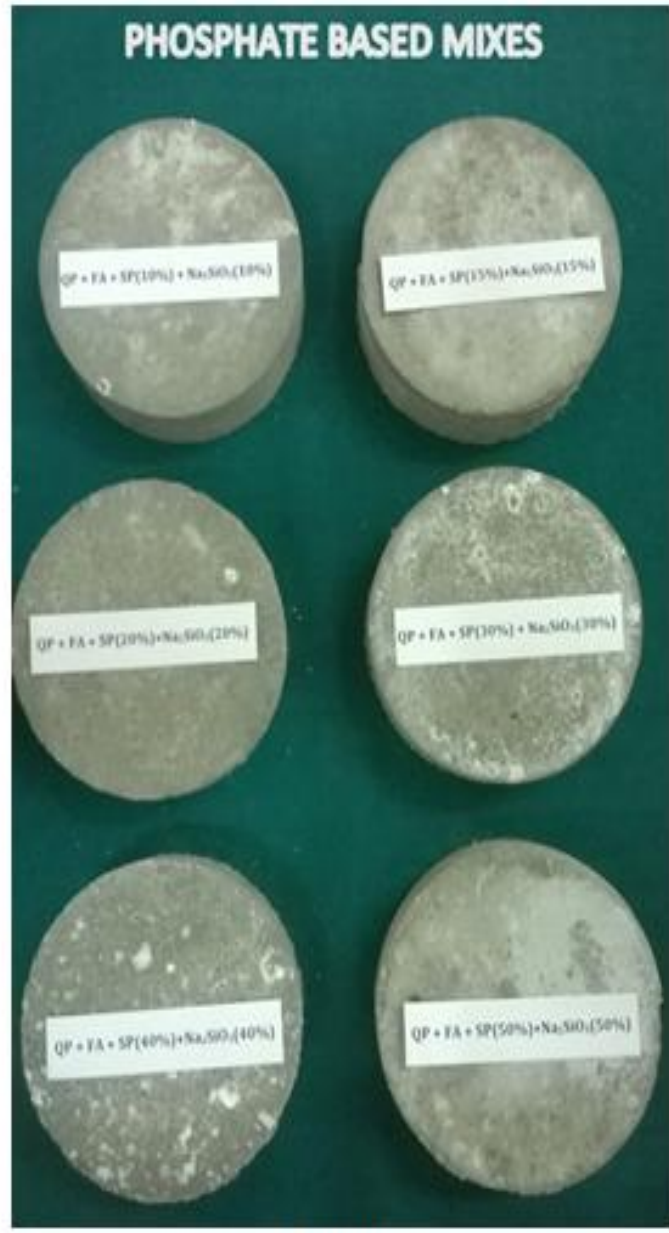

Fig 6 


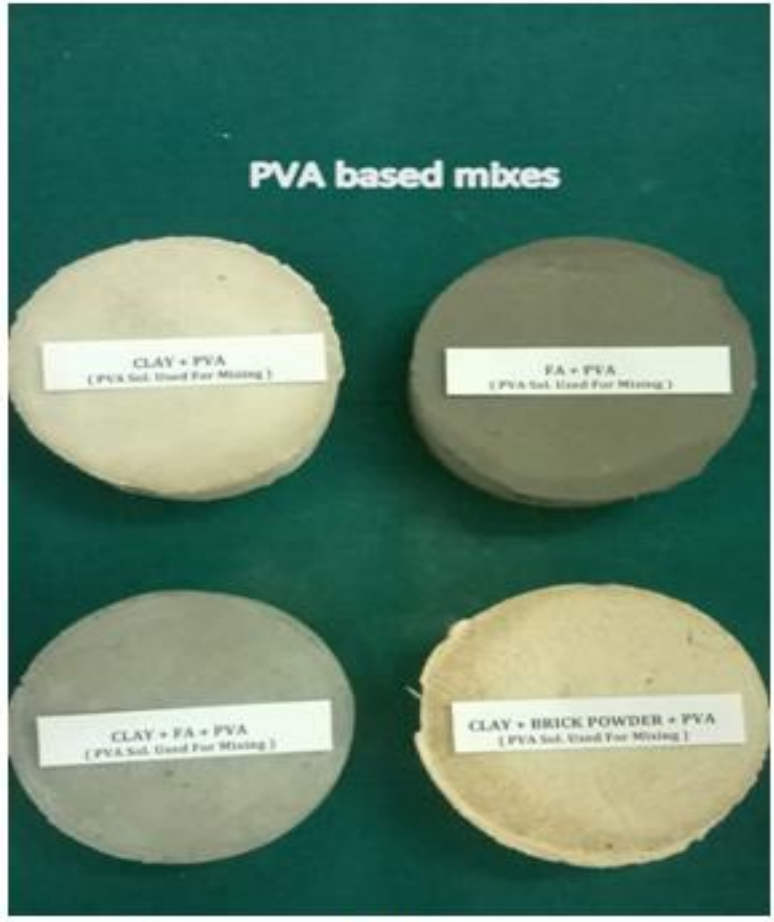

Fig 7

Table -1:Preliminary study report on Alternate Binders with Composition

\begin{tabular}{|c|c|c|c|c|c|c|}
\hline $\begin{array}{l}\text { S. } \\
\text { No }\end{array}$ & Composition & $\begin{array}{c}\text { Dry } \\
\text { Density }\end{array}$ & Color & $\begin{array}{c}\text { Nail } \\
\text { penetration }\end{array}$ & $\begin{array}{c}\text { Lateral } \\
\text { shrinlvage }\end{array}$ & Observations \\
\hline 1. & $\begin{array}{c}\mathrm{QP}+\mathrm{FA}+ \\
\mathrm{SP}(10 \%)+\mathrm{Na}_{2} \mathrm{SiO}_{3}(10 \%)\end{array}$ & 1.64 & Grey & Hard & NII. & $\begin{array}{l}\text { Very slight leaching of } \\
\text { phosphate on top surface. } \\
\text { Light in weight. }\end{array}$ \\
\hline 2. & $\begin{array}{c}\mathrm{QP}+\mathrm{FA}+ \\
\mathrm{SP}(15 \%)+\mathrm{Na}_{2} \mathrm{SiO}_{3}(15 \%)\end{array}$ & 1.61 & Grey & Hard & NII & $\begin{array}{l}\text { Moderate leaching of } \\
\text { phosphates on top } \\
\text { surface. Light in weight. }\end{array}$ \\
\hline 3. & $\begin{array}{c}\mathrm{QP}+\mathrm{FA}+ \\
\mathrm{SP}(20 \%)+\mathrm{Na}_{2} \mathrm{SiO}_{3}(20 \%)\end{array}$ & 1.65 & Grey & Hard & 1.2048 & $\begin{array}{c}\text { Moderate leaching of } \\
\text { phosphates on top surface. } \\
\text { Light in weight. }\end{array}$ \\
\hline 4. & $\begin{array}{c}\mathrm{QP}+\mathrm{FA}+ \\
\mathrm{SP}(30 \%)+\mathrm{Na}_{2} \mathrm{SiO}_{3}(30 \%)\end{array}$ & 1.58 & Grey & Hard & 1.8072 & $\begin{array}{l}\text { Moderate leaching of } \\
\text { phosphates on top surface. } \\
\text { Light in weight. }\end{array}$ \\
\hline 5. & $\begin{array}{c}\mathrm{QP}+\mathrm{FA}+ \\
\mathrm{SP}(40 \%)+\mathrm{Na}_{2} \mathrm{SiO}_{3}(40 \%)\end{array}$ & 1.54 & Grey & Hard & NII & $\begin{array}{c}\text { More leaching and } \\
\text { crystallization of } \\
\text { phosphate on top surface. }\end{array}$ \\
\hline 6. & $\begin{array}{c}\mathrm{QP}+\mathrm{FA}+ \\
\mathrm{SP}(50 \%)+\mathrm{Na}_{2} \mathrm{SiO}_{3}(50 \%)\end{array}$ & 1.30 & Grey & Soft & NII & $\begin{array}{c}\text { Leaching and } \\
\text { crystallization; } \\
\text { dislodgement of particles } \\
\text { observed. }\end{array}$ \\
\hline 7. & $\begin{array}{c}\mathrm{FA}+\mathrm{SAND}+\mathrm{Na}_{2} \mathrm{CO}_{3}+\mathrm{CaCO}_{3} \\
\left(\mathrm{Na}_{2} \mathrm{CO}_{3}, \mathrm{Ca}_{2} \mathrm{CO}_{3} \text { in 1:1 ratio }\right)\end{array}$ & 1.66 & Grey & Soft & NII & $\begin{array}{l}\text { Heavy Leaching and } \\
\text { crystallization of } \\
\text { Carbonates; dislodgement } \\
\text { of particles observed. }\end{array}$ \\
\hline 8. & $\begin{array}{c}\mathrm{FA}+\mathrm{SAND}+\mathrm{Na}_{2} \mathrm{CO}_{3}+\mathrm{CaCO}_{3} \\
\left(\mathrm{Na}_{2} \mathrm{CO}_{3}, \mathrm{Ca}_{2} \mathrm{CO}_{3} \text { in } 2: 1 \text { ratio }\right)\end{array}$ & 1.87 & Grey & V. Hard & NII & $\begin{array}{c}\text { Very hard mass yet excess } \\
\text { leaching of carbonates and } \\
\text { cystallization. }\end{array}$ \\
\hline 9. & $\mathrm{FA}+\mathrm{Na}_{2} \mathrm{CO}_{3}+\mathrm{CaCO}_{3}$ & 1.45 & Grey & V. Hard & NII. & $\begin{array}{l}\text { Hard mass yet excess } \\
\text { leaching of carbonates and } \\
\text { cystallization, } \\
\text { dislodgement occurred in } \\
\text { top peripheral line. }\end{array}$ \\
\hline 10. & $\mathrm{FA}+\mathrm{SAND}+\mathrm{MgO}+\mathrm{MgCl}_{2}$ & 1.79 & Grey & V. Hard & NIIL & $\begin{array}{l}\text { Smooth, shiny, cracli-free } \\
\text { and very hard mass. }\end{array}$ \\
\hline 11. & $\mathrm{SAND}+\mathrm{MgO}+\mathrm{MgCl}_{2}$ & 1.85 & $\begin{array}{l}\text { sand } \\
\text { color }\end{array}$ & V. Hard & 2.2096 & $\begin{array}{l}\text { Smooth, shiny, cracli-free } \\
\text { and verv hard mass; slight } \\
\text { leaching of salts at the } \\
\text { sides and bottom surface. }\end{array}$ \\
\hline 12. & $\mathrm{FA}+\mathrm{SAND}_{+} \mathrm{MgO}_{+} \mathrm{MgSO}_{4}$ & 1.90 & Grey & V. Hard & NII & $\begin{array}{l}\text { Very hard, no crack, } \\
\text { leaching and disbandment. }\end{array}$ \\
\hline 13. & $\begin{array}{c}\mathrm{FA}+\mathrm{SAND}+\mathrm{MgO}+\mathrm{MgPO}_{4}+\mathrm{A} . \mathrm{ACID} \\
\left(\mathrm{A} . \mathrm{ACID}-\mathrm{C}_{3} \mathrm{H}_{4} \mathrm{O}_{2}\right)\end{array}$ & 1.67 & Grey & V. Hard & NII & $\begin{array}{l}\text { Very hard, no crack, } \\
\text { leaching and disbandment. }\end{array}$ \\
\hline 14. & $\mathrm{FA}+\mathrm{SAND}+\mathrm{MgO}+\mathrm{LATEX}+\mathrm{H}_{2} \mathrm{O}_{2}$ & 1.54 & Grey & V. Hard & NII & $\begin{array}{l}\text { Random spot leaching of } \\
\text { MgO found on top } \\
\text { surface; slightly glossy. }\end{array}$ \\
\hline 15. & $\begin{array}{c}\mathrm{FA}+\mathrm{SAND}+\mathrm{MgO}+\mathrm{MgCl}_{2}+\mathrm{P} \cdot \mathrm{ACID} \\
\left(\text { P.ACID }-\mathrm{C}_{6} \mathrm{H}_{6} \mathrm{O}_{2} \text { ) }\right.\end{array}$ & 1.34 & Grey & Soft & NII & $\begin{array}{l}\text { Dislodgement of particles, } \\
\text { less compaction }\end{array}$ \\
\hline
\end{tabular}




\begin{tabular}{|c|c|c|c|c|c|c|}
\hline 16. & $\begin{array}{c}\text { FA + DAP BINDER } \\
\text { (DAP BINDER Used For Mixing) }\end{array}$ & 1.34 & Grey & Soft & 1.2048 & $\begin{array}{l}\text { No hardmess, not resisting } \\
\text { nail penetration }\end{array}$ \\
\hline 17. & $\begin{array}{c}\text { CLAY + DAP BINDER } \\
\text { (DAP BINDER Used For Miving) }\end{array}$ & 1.82 & $\begin{array}{l}\text { Dark } \\
\text { Brown }\end{array}$ & VHard & 9.6386 & $\begin{array}{l}\text { Very hard Metallic soumd. } \\
\text { Highly cohesive, excess } \\
\text { shrinkage. }\end{array}$ \\
\hline 18. & $\begin{array}{l}\text { FA + CLAY + DAP BINDER } \\
\text { (DAP BINDER Used For Mixing) }\end{array}$ & 1.53 & Brown & Soft & 1.8072 & $\begin{array}{l}\text { Moderate shrinkage. Soft, } \\
\text { mild popouts observed on } \\
\text { the finished surface. }\end{array}$ \\
\hline 19. & $\begin{array}{l}\text { FA + SAND + DAP BINDER } \\
\text { (DAP BINDER Used For Miving) }\end{array}$ & 1.63 & Grey & Soft & NII & $\begin{array}{l}\text { Soft, no cracks but mild } \\
\text { leaching observed }\end{array}$ \\
\hline 20. & $\begin{array}{l}\text { SAND + CLAY + DAP BINDER } \\
\text { (DAP BINDER Used For Mixing) }\end{array}$ & 2.04 & Brown & VHard & 6.0241 & $\begin{array}{l}\text { Very hard, stone like mass, } \\
\text { no cracks or leaching; high } \\
\text { shrinkage. }\end{array}$ \\
\hline 21. & $\begin{array}{l}\text { FA+ SAND + CLAY + DAP BINDER } \\
\text { (DAP BINDER Used For Mixing) }\end{array}$ & 1.87 & $\begin{array}{l}\text { Light } \\
\text { Brown }\end{array}$ & Hard & 4.2169 & $\begin{array}{l}\text { Moderately hard; mild } \\
\text { shrinkage, no cracks or } \\
\text { leaching. }\end{array}$ \\
\hline 22. & FA + BORAX & 1.50 & Grey & Soft & NII & $\begin{array}{l}\text { Soft material, yet } \\
\text { maintained shape and size } \\
\text { without cracks or leaching. }\end{array}$ \\
\hline 23. & CLAY + BORAX & 1.97 & $\begin{array}{l}\text { Dark } \\
\text { brown }\end{array}$ & VHard & 12.6506 & $\begin{array}{l}\text { Very strong, high shrinkage. } \\
\text { No cracks or leaching. }\end{array}$ \\
\hline 24. & FA + SAND + CLAY + BORAX & 1.76 & Brown & Soft & 2.4096 & $\begin{array}{c}\text { Soft material. No cracks or } \\
\text { shrinkage. }\end{array}$ \\
\hline 25. & SAND + W.CLAY + BORAX & 1.90 & $\begin{array}{l}\text { Very } \\
\text { Light } \\
\text { brown }\end{array}$ & Hard & 2.4096 & $\begin{array}{c}\text { Moderately hard No } \\
\text { shrinkage but slight } \\
\text { shrinkage. }\end{array}$ \\
\hline 26. & $\begin{array}{c}\text { W.CL.AY + G.P } \\
\text { (G.P Sol. Used For Mixing) }\end{array}$ & 1.64 & $\begin{array}{c}\text { Off- } \\
\text { white }\end{array}$ & VHard & 7.2289 & $\begin{array}{l}\text { Very hard mass like stone, } \\
\text { no cracks or shrinkage or } \\
\text { leaching. }\end{array}$ \\
\hline 27. & $\begin{array}{c}\text { FA + G.P } \\
\text { (G.P Sol. Used For Mixing) }\end{array}$ & 1.47 & Grey & VHard & 1.8072 & $\begin{array}{l}\text { Very hard mass like stcoe, } \\
\text { no cracks or shrinkage or } \\
\text { leaching. }\end{array}$ \\
\hline 28. & $\begin{array}{l}\text { W.CLAAY + FA + SAND + G.P } \\
\text { (G.P Sol. Used For Mixing) }\end{array}$ & 1.79 & $\begin{array}{l}\text { Light } \\
\text { Grey }\end{array}$ & VHard & 1.2048 & $\begin{array}{l}\text { Very hard mass like stcue, } \\
\text { no cracks or shrinkage or } \\
\text { leaching. }\end{array}$ \\
\hline 29. & $\begin{array}{l}\text { W.CLAAY + FA + SAND + G.P } \\
\text { ( G.P Sol. Used For Miving) }\end{array}$ & 1.73 & $\begin{array}{l}\text { Brown } \\
\text {-ish } \\
\text { grey }\end{array}$ & VHard & NII & $\begin{array}{l}\text { Very hard mass like stoue, } \\
\text { no cracks or shrinkage or } \\
\text { leaching. }\end{array}$ \\
\hline 30. & $\begin{array}{c}\text { F.A + BRICK POWDER + G.P } \\
\text { ( G.P Sol. Used For Miving) }\end{array}$ & 1.91 & $\begin{array}{c}\text { Off- } \\
\text { white }\end{array}$ & Hard & 3.6145 & $\begin{array}{l}\text { Very hard mass, no cracks } \\
\text { or shrinkage or leaching. }\end{array}$ \\
\hline 31. & W.CLAY + PVA & 1.45 & Grey & VHard & NII & $\begin{array}{l}\text { Stone like mass, moderately } \\
\text { strong, no cracks, Requires }\end{array}$ \\
\hline 32. & $\begin{array}{c}\text { W.CLAY+ FA + PVA } \\
\text { (PVA Sol. Used For Mixing) }\end{array}$ & 2.01 & $\begin{array}{l}\text { Light } \\
\text { gey }\end{array}$ & VHard & 4.2169 & $\begin{array}{l}\text { Stone like mass, moderately } \\
\text { strong, no cracks, slight } \\
\text { shrinkage. }\end{array}$ \\
\hline 33. & $\begin{array}{l}\text { W.CLAY+BRICK POWDER+ PVA } \\
\text { (PVA Sol. Used For Mixing) }\end{array}$ & 1.89 & $\begin{array}{l}\text { Light } \\
\text { brown }\end{array}$ & Hard & 5.4200 & $\begin{array}{l}\text { Moderately strong, slight } \\
\text { shrinkage, no cracks. }\end{array}$ \\
\hline
\end{tabular}

\section{Results and Discussions}

The result are tabulated in table 1 for all 33 combinations with due observations. From the observations it is clear that some specimens have showed leaching and crystallization of salts used particularly, in specimens containing phosphates and of Carbonates. This indicates the need for optimization of mixes. Also it is observed many compositions observed were of stone like mass in 24 hours and therefore these materials are promising as future concrete. Wherever clay was used, specimen showed shrinkages, this indicates the need of use of shrinkage compensators.

\section{Conclusions:}

1. All of the 33 cast specimens were eco-friendly, economical and low cost materials.

2. All the specimens set in 12- 24 hours; some phosphate based mixes exhibited flash setting.

3. The mixes obtained with clay particularly exhibits high cohesion and therefore, loss in slump and moderate to excessive shrinkage.

4. Many mixes as indicated had been exhibited metallic sound.

5. All these mortars developed are suitable for structural applications, general repair works, also for treating the corroded areas.

6. Also, most of the mixes obtained are of good aesthetic appeal.

\section{References}


[1]. A.M. Neville, "Properties of concrete", $4^{\text {th }}$ Edition,Pearson Education(Singapore) Pvt. Ltd(2004)

[2]. P.K.Mehta and P.J.M. Monteiro, "Concrete Microstructure, Properties and Material", Indian Concrete Institute(1999)

[3]. W.K.W.Lee and J.S.J Van Deventer, Chemical interactions between siliceous aggregates and low Ca-alkali activated cements. Cem Concr Res37(2007)844-855

[4]. P.Durson and J.L.Provis. Designing Precursors for geopolymer J.Ann Ceram Soc 91(2008)3864-3869

[5]. E.M.Gartner,Industrially interesting approaches to" low $\mathrm{CO}_{2}$ " cements, Cem Concr Res34(2004)1489-1498

[6]. Alternatives to Portland cement : An Introduction Practical action, The Schumacher centre for Technology and Development, Bourton Hall, Bourton on Dunsmore Rugby CV23 9Z,UK

[7]. Manjit Singh, M.Garg and S.S .Rehsi, Durability of Phospho-gypsum based water-resistant anhydrite binder, Cem Concr Res20(1990)271-276

[8]. E.M.Gartner and D.E Masphee,A physic- chemical basis for novel cementitious binders,Cem Concr Res41(2011)736-749

[9]. K.Quillin, Calcium Sulpho-aluminates cements $\mathrm{CO}_{2}$ reduction, Concrete properties and applications, BRE Report(Garston , UK),BR 496-2007

[10]. L.J.Vandeperre, M.Liska and A.Al-Tabaa, Hydration and mechanical properties of magnesia, pulverized fuel ash, and Portland cement blends, J.Q Materials in Civil Engg.20(2008) 375-383

[11]. E.Benhelal, A.Rafiei and E.Shamsaei,Green cement production: Potentials and Achievements, Intl. J. of Chemical Engg. and Application 3(2012)407-409

[12]. J.Cross and J.Stephens, An alternative to Portland cement concrete Proceed, Third Intl. cement as construction materials: Pertama New, Innovations and Structural Implications, Vancouver Be, August 22-24,2005 